\title{
Ultrastructural Observations on Cellular and Subcellular Aspects of Experimental Mycoplasma pneumoniae Disease
}

\author{
JOHNNY L. CARSON,* ALBERT M. COLLIER, AND SHIH-CHIN S. HU \\ Infectious Disease Division, Department of Pediatrics and Frank Porter Graham Child Development \\ Center, University of North Carolina, Chapel Hill, North Carolina 27514
}

\begin{abstract}
The ultrastructural organization of Mycoplasma pneumoniae membranes and spatial relationships of this pathogen to epithelial cells in tracheal organ cultures were examined ultrastructurally by freeze-fracture techniques. Areas of morphologically distinct cell membrane variability characterized by membrane blebs and altered distributions of membrane associated particles were observed in replicas of $M$. pneumoniae cells. Inspection of the host tracheal epithelium demonstrated the alignment of $M$. pneumoniae to the epithelium with an accompanying deterioration in the integrity of the lumenal surface membranes and subsequent loss of the epithelial cell cytosol. Ciliary dysfunction was suggested by the observation of ciliary lesions and of disorganized epithelial cell cilia. The methodology used in these studies has permitted a new perspective of host-pathogen interactions at both the cellular and subcellular levels in tracheal organ cultures. These studies may also illustrate ultrastructural correlates of the alteration of host macromolecular synthesis in experimental $M$. pneumoniae infection.
\end{abstract}

Mycoplasma pneumoniae is a respiratory pathogen of humans which can be responsible for a variety of respiratory diseases ranging from upper respiratory infections, bronchitis, and tracheitis to primary atypical pneumonia (9). Previous studies of $M$. pneumoniae in tracheal organ cultures and in clinical specimens have demonstrated the presence of a highly specialized terminal organelle $(5-7,18)$ by which the organism appears to orient and attach in a specific manner to the host cell surface $(5,7,8,13$, $15,18)$. These studies have also provided information regarding the biochemical composition of $M$. pneumoniae (18) and aspects of host cell injury such as ciliostasis and altered cellular metabolism in experimental infection.

Freeze-etch studies of other mycoplasma species $(1,11,16,17)$ have demonstrated the presence of membrane blebs, membrane-associated particles thought to represent primarily proteinaceous elements of the membrane, and particle clearing on fractured membrane faces. In this study, the use of the freeze-fracture technique (14) has provided some additional insights into the structure and possible mechanisms of $\boldsymbol{M}$. pneumoniae not attainable by conventional ultrastructural' methods. The resulting morphological observations are consistent with biochemical data in experimental $M$. pneumoniae infection.

\section{MATERIALS AND METHODS}

Preparation of tracheal organ cultures. Tracheal organ cultures were prepared by the methods previously described by Collier and Baseman (6) using 80- to 100-g male Syrian hamsters. A slight modification of this technique was employed for freeze-fracture specimens to facilitate sample preparation (see below). Briefly, the trachea, rather than being cut into rings, was sliced longitudinally after resection. The tracheal slices were subsequently maintained in the same manner as described for tracheal rings.

Mycoplasma culture. A log phase culture of virulent $M$. pneumoniae M129-B16 or M129-B19 in a standard mycoplasma broth (12) was used to infect the tracheal organ cultures. The seventh broth passage of this strain (M129-B7) is maintained as no. 29342 by the American Type Culture Collection.

Experimental infection. Freshly prepared tracheal explants were inoculated with ca. $2.5 \times 10^{8}$ colony-forming units (CFU) of M129-B16 or $4.25 \times 10^{8}$ CFU of M. pneumoniae M129-B19 in $1.0 \mathrm{ml}$ of broth for $1 \mathrm{~h}$. Infected and control cultures were subsequently incubated in ca. $5 \mathrm{ml}$ of additional broth at $37^{\circ} \mathrm{C}$ with $5 \% \mathrm{CO}_{2}$ in air for 24 and $48 \mathrm{~h}$ before fixation.

Electron microscopy techniques. After incubation, all tracheal explants were removed from the culture dishes and fixed in $2 \%$ glutaraldehyde- $2 \%$ paraformaldehyde in $0.1 \mathrm{M}$ phosphate buffer. The tracheal slices required the removal of the epithelial cell layer for freeze-etching and freeze-fracturing to exclude cartilage and connective tissue from the specimens. This was done by carefully dissecting the epithelial cell matrix of infected and control specimens away from the longitudinally sliced trachea using a pair of fine grade forceps. The isolated epithelium was subsequently rinsed in $0.1 \mathrm{M}$ phosphate buffer. Before freezing, the tissue was treated with a cryoprotectant consisting of $25 \%$ glycerol in $0.1 \mathrm{M}$ phosphate buffer for $1 \mathrm{~h}$. Pieces of the epithelial cell matrix were positioned on gold freeze-etch specimen stubs or double 
replica mounts and frozen in Freon 22 with subsequent rapid transfer to a holding Dewar flask of liquid nitrogen. Freeze-etching and -fracturing cycles were carried out in a Balzers M360 freeze-etch instrument (Balzers High Vacuum Corp., Nashua, N.H.) at a stage temperature of $-100^{\circ} \mathrm{C}$ and sublimation time of $2 \mathrm{~min}$ for etched specimens. The specimens were shadowed with platinum-carbon and coated with carbon. The replicas were cleaned in a solution of $5 \%$ sodium dichromate in $50 \% \mathrm{H}_{2} \mathrm{SO}_{4}$ and retrieved from distilled water onto 300-mesh copper grids. Replicas were viewed with a Zeiss EM-10A electron microscope at an accelerating voltage of $60 \mathrm{kV}$.

\section{RESULTS}

Tracheal organ cultures provided an excellent model system for the ultrastructural study of the cellular detail of mycoplasma cells as well as their interactions with host tracheal epithelial cells. Cells of $M$. pneumoniae observed in these preparations were generally very pleomorphic. The cells appeared to conform to physical spaces imposed by the presence of other cells, resulting in microcolonies with cells which were highly contorted about one another. Although great variability in cell shape and size was noted, many cells demonstrated rounded, pear-shaped, or lobulated cell bodies ranging from 1.0 to $2.0 \mu \mathrm{m}$ in length and 0.1 to $0.3 \mu \mathrm{m}$ in width (Fig. 1).

Closer observation of freeze-fracture replicas revealed several unique features of the $M$. pneumoniae cell membranes. These preparations demonstrated the presence of membrane associated particles ca. $10 \mathrm{~nm}$ in diameter over the hydrophobic fracture face of the cell membrane (Fig. 1). These particles were generally more numerous in convex (P-face) membrane fractures than in concave (E-face) fractures (The designation of $\mathrm{E}$-face and $\mathrm{P}$-face in freeze-fractured membranes is based on the generally accepted premise that the fracture plane preferentially passes through the hydrophobic lipid bilayer of biological membranes. This results in two membrane fracture faces, one a concave face associated with the extracellular aspect of the cell [E-face or EF], and the other a convex face [P-face or PF] associated with the cytoplasmic

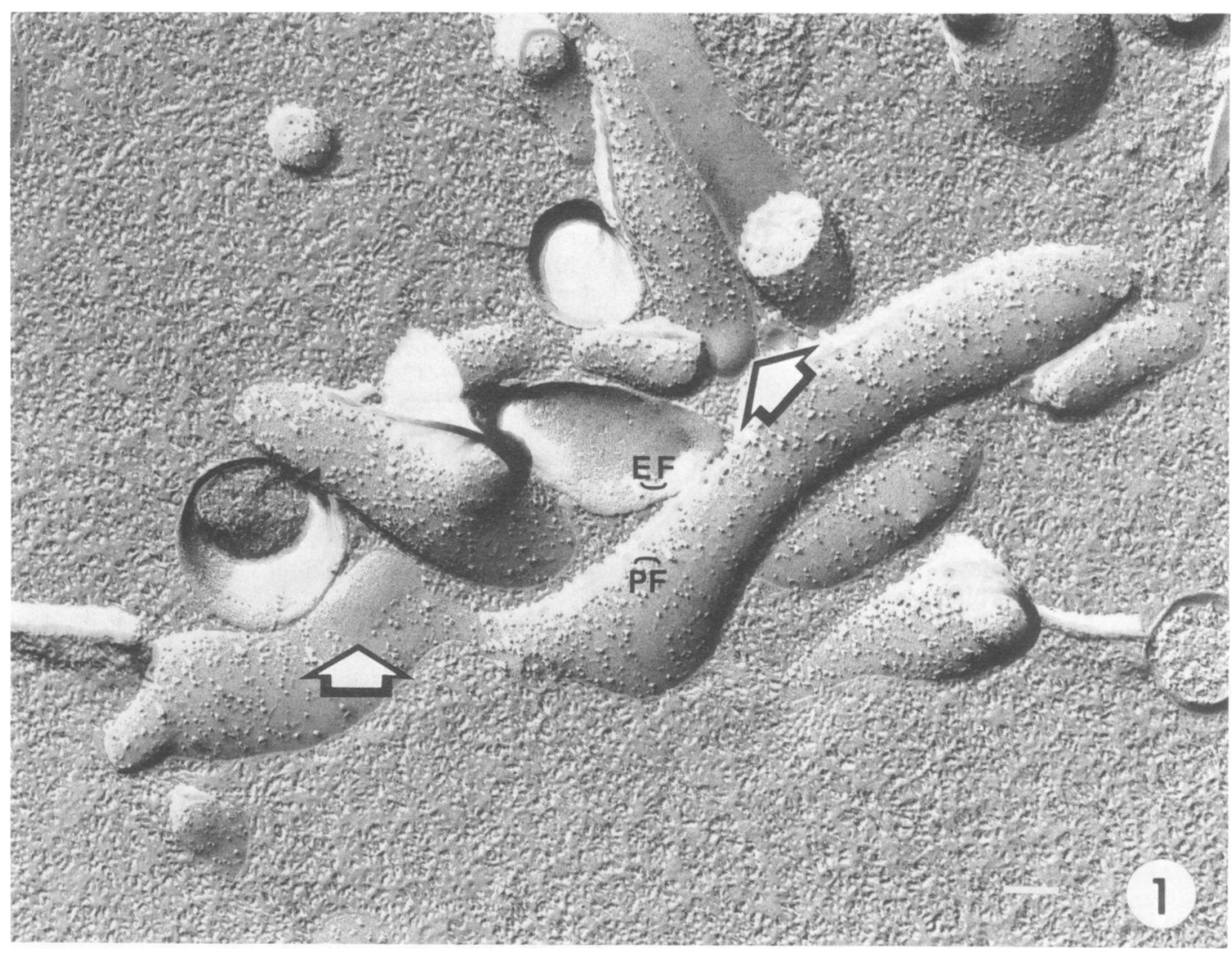

Fig. 1. A freeze-etch preparation illustrating several M. pneumoniae cells. Areas of particle clearing and particle-free blebs can be seen on some of these cells (arrows). Also illustrated are $P$-face (PF) convex and $E$ face $(E F)$ concave fractures of the mycoplasma cell membranes. Bar, $0.1 \mu \mathrm{m}$. 
aspect of the cell [2].). P-face fractures of the mycoplasma membranes occasionally illustrated clearly delineated particle-free zones and blebs (Fig. 1 and 2). The particle-free blebs generally were observed to protrude outward from the cell surface a distance of ca. $0.1 \mu \mathrm{m}$; however, larger, more irregular blebs were noted as well as nonprotruding particle-free zones on the fractured membrane face. These particle-free blebs have been observed when the cells were in close proximity to the membrane of the host tracheal epithelial cells (Fig. 2).

Inspection of freeze-etch replicas also illustrated the ultrastructure of tracheal epithelium in control organ cultures, an observation which has been described comprehensively elsewhere (4). Freeze-fracture preparations of the infected organ cultures revealed microcolonies, as well as individual $M$. pneumoniae cells, closely associated with the host epithelial cell surfaces (Fig. 2 to 5). Identification of infecting mycoplasma

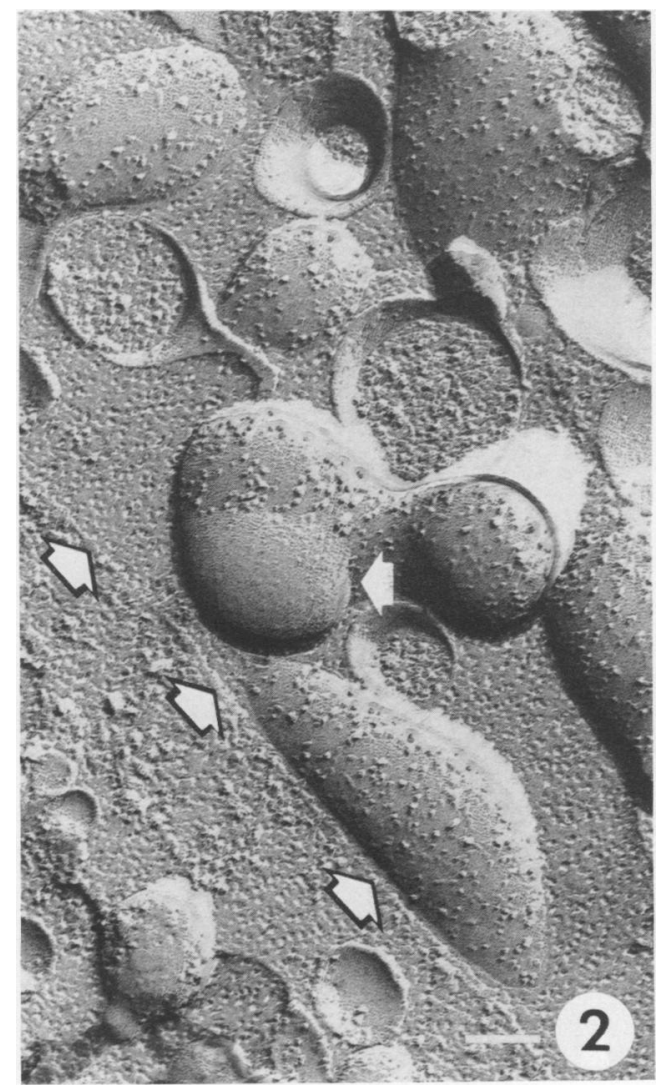

Fig. 2. Freeze-etch preparation illustrating a particle-free bleb of M. pneumoniae (white arrow) in close proximity to the tracheal epithelial cell membrane in organ culture (white on black arrows). Bar, $0.1 \mu \mathrm{m}$. cells was based on several criteria; namely, their position in relation to the epithelial cell surface, their pleomorphic structure, the complement of membrane-associated particles revealed in membrane fractures, and by the presence of particle-free zones and blebs observed in fractures through the lipid bilayer of the mycoplasma membrane. In addition to attachment of individual mycoplasma cells between cilia and microvilli, M. pneumoniae cells also demonstrated an affinity for focal attachment at epithelial intercellular borders in an ordered rosette fashion or were observed closely associated with the host cell surface as amorphous clusters of cells (Fig. 3 and 4). The fracture plane passing through the cytoplasm of a mycoplasma cell at the point of attachment to the tracheal epithelium also revealed a shadowed core consistent in position with the terminal organelle, which can be seen in ultrathin sections of $M$. pneumoniae (Fig. 5). Freeze-fracture preparations of $M$. pneumoniae-infected tracheal epithelium also revealed a marked deterioration in the integrity of the host epithelial cell membrane (Fig. 3 and 6). The deterioration and rupture of the epithelial cell membranes appeared in the freeze-fracture preparations as a frothy interface between the infected cells and the extracellular milieu of the specimen, often with the leakage of the

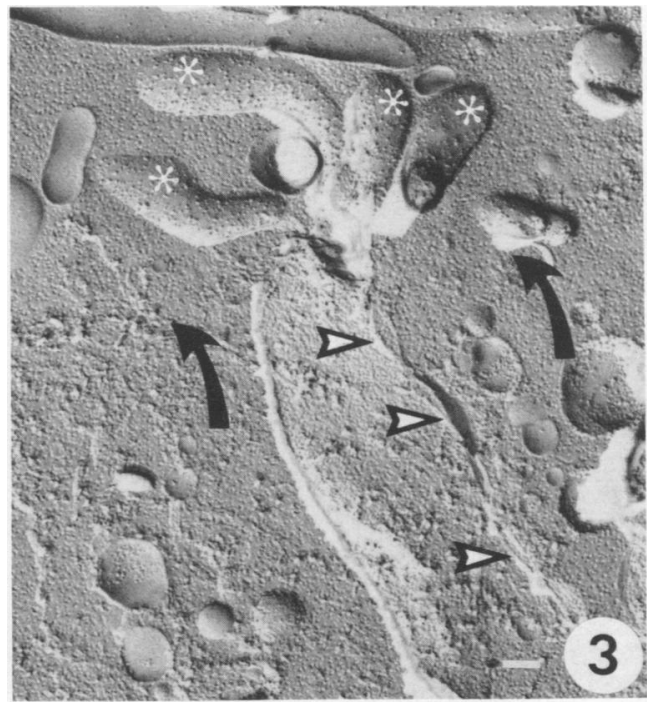

Fig. 3. Electron micrograph illustrating a rosette of mycoplasma cells $\left(^{*}\right)$ adhering to a focal lumenal intercellular site between two epithelial cells. The curved arrows indicate areas approximating the deteriorating lumenal surface of the epithelial cell membrane. The small arrows indicate an intact lateral border between two adjacent epithelial cells. Bar, 0.1 $\mu m$. 
cytosol from the cell.

A prominent ultrastructural aspect of cellular injury reconfirmed by the present studies was the apparent effect of this pathogen on the intramembranous particles of the epithelial cell cilia. Primarily, the molecular complex known as the ciliary necklace, which occurs at the base of the epithelial cell cilia (10), appeared to be markedly altered by infection with $M$. pneumoniae (Fig. 7 and 8). Roughly organized remnants of the ciliary necklace were frequently observed in the affected cells, but the general view was one of complete disorganization of this ultrastructural complex. Additionally, ciliary dysfunction was suggested by a general disorganization of the cilia of infected cells (Fig. 4 and 8 ). Also, plaques of membrane-associated particles were observed on the shafts of the affected cilia in contrast to a random uniform distribution of these particles on the ciliary shafts in control cultures. The individual membrane-associated particles comprising these plaques were ca. 10 $\mathrm{nm}$ in diameter. The size of individual plaques varied with the number of particles, most

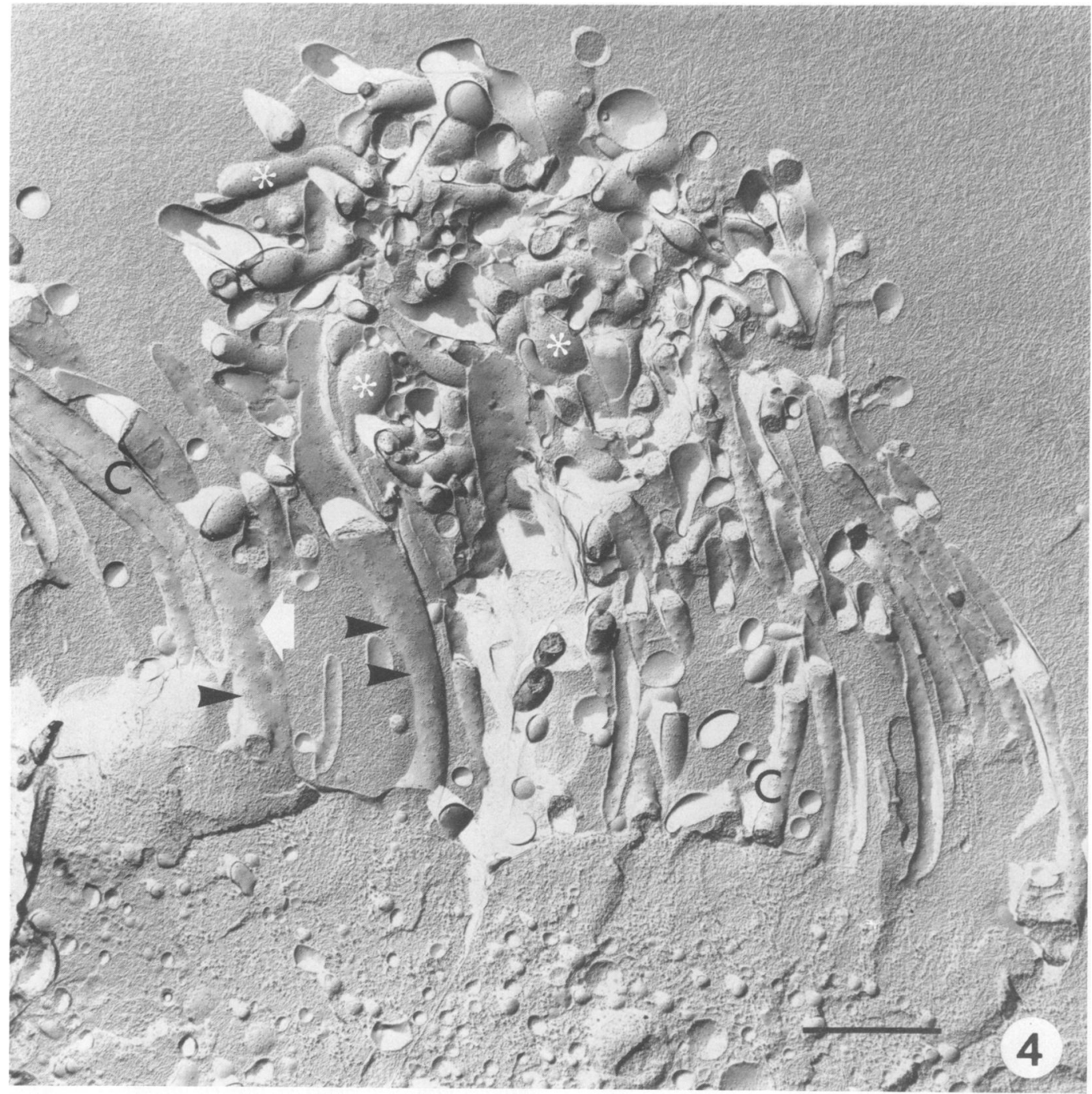

Fig. 4. Electron micrograph illustrating a microcolony of $M$. pneumoniae associated with host tracheal epithelium in organ culture in which the structure of the host epithelial cells can still be discerned. Note also the numerous pleomorphic mycoplasma cells $\left({ }^{*}\right)$ interspersed among the epithelial cell cilia (C), clumping of the cilia (white arrow), and the plaques of membrane-associated particles on the ciliary shafts (black arrows). Bar, $1.0 \mu \mathrm{m}$. 


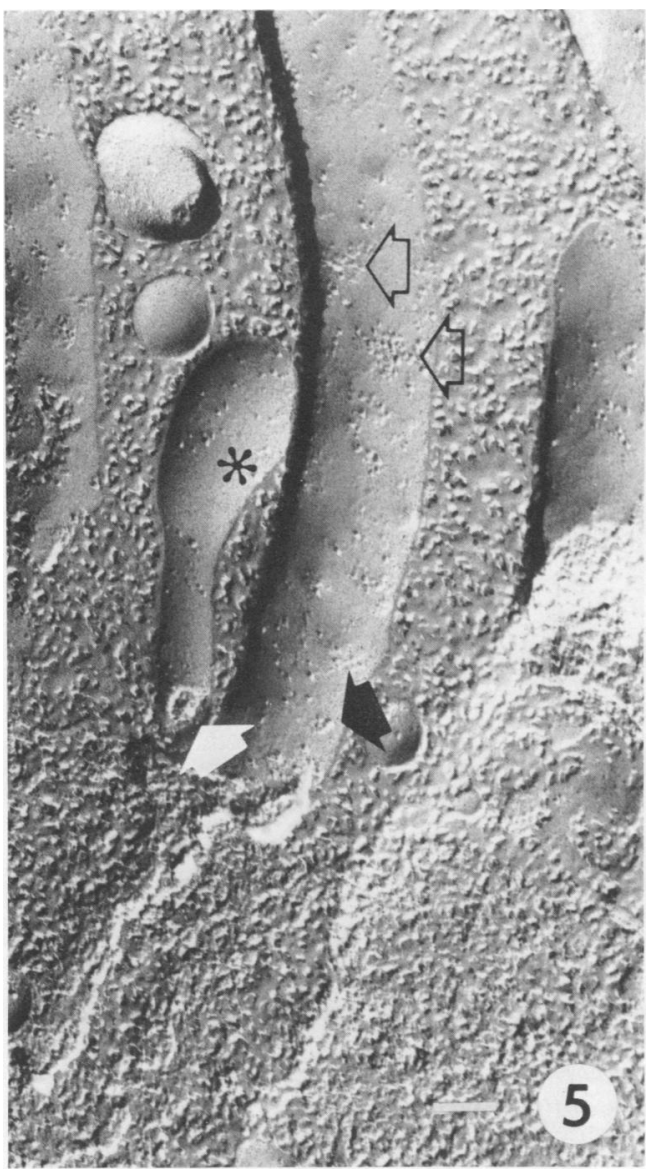

Fig. 5. High magnification electron micrograph illustrating a mycoplasma cell $\left(^{*}\right)$ attached to the host epithelial cell surface at the base of a cilium. The cilium clearly illustrates the loss of the ciliary necklace at its base (closed black arrow) and plaques of membrane-associated particles on its shaft (open black arrows). The fracture plane shown in this micrograph extended through the tip of the mycoplasma cell and subsequent shadowing revealed a central core in the attachment area (white arrow). Bar, 0.1 $\mu m$.

plaques being comprised of from 2 to 20 or more directly apposing particles.

\section{DISCUSSION}

The ultrastructural views provided by freezefracture of $M$. pneumoniae-infected tracheal organ cultures have provided a unique ultrastructural perspective of the cellular and subcellular biology of host-parasite interactions. This approach has been particularly advantageous in assessing cell membrane responses of both host and pathogen in experimental infection. Also, facets of the ultrastructure of $M$. pneumoniae which may be reflective of the growth, metabolism, and/or pathogenicity of this organism, as well as responses of host target cells to this infectious agent, have been revealed.

In freeze-fracture preparations, membrane-associated particles were observed on all membrane fracture faces of the mycoplasma cells, but were generally more abundant on the convex $P$-face than on the concave E-face of the fractured membrane. These particles are generally accepted as representing integral membrane proteins closely associated with the internal aspect of the cell membrane (17).

Another noteworthy feature of $M$. pneumoniae cells observed in freeze-fracture preparations has been the observation of particle-free zones and blebs on the fractured membrane surface. In previous ultrastructural studies on $M$. gallisepticum it was observed that newly formed blebs of the cell membrane possess a smooth texture, whereas older, more mature membrane blebs illustrate a roughened appearance (1). Particle clearing in $M$. pneumoniae membranes may indicate areas of rapid expansion, membrane biogenesis, or specialized transitional membrane pools specifically primed to assist in recognition and attachment to the host epithelial cell. Although particle-free blebs of $M$. pneumoniae are observed in close proximity to the host epithelial cell membrane, other closely aligned mycoplasma cells appear with a normal complement of membrane-associated particles, suggesting that such areas are a temporal phase of membrane structure, thus imparting a dynamic, transitional character to the mycoplasma membrane. It is clear from other electron microscopic studies that the specialized cytoplasmic fibril observed in ultrathin sections of $M$. pneumoniae is also involved in the orientation and attachment of the organism to the host cell surface $(5-7,18)$. It follows that areas of the cell membrane may also be specialized structurally and functionally for host recognition and attachment. These observations thus suggest the existence of fibril-membrane complexes, which may provide continuity between cytoplasmic and cell membrane elements of the mycoplasma cell involved in attachment processes. Such complexes may be of particular significance in the positioning and orientation of the mycoplasma cell to the host cell surface via mechanisms such as chemotaxis, alterations of membrane permeability, and/or mechanical contact.

The response of the host cell membrane to experimental infection by $M$. pneumoniae revealed in this study represents facets of pathogenesis not previously recognized. The epithelial cell cilia are sites of ultrastructural lesions indi- 


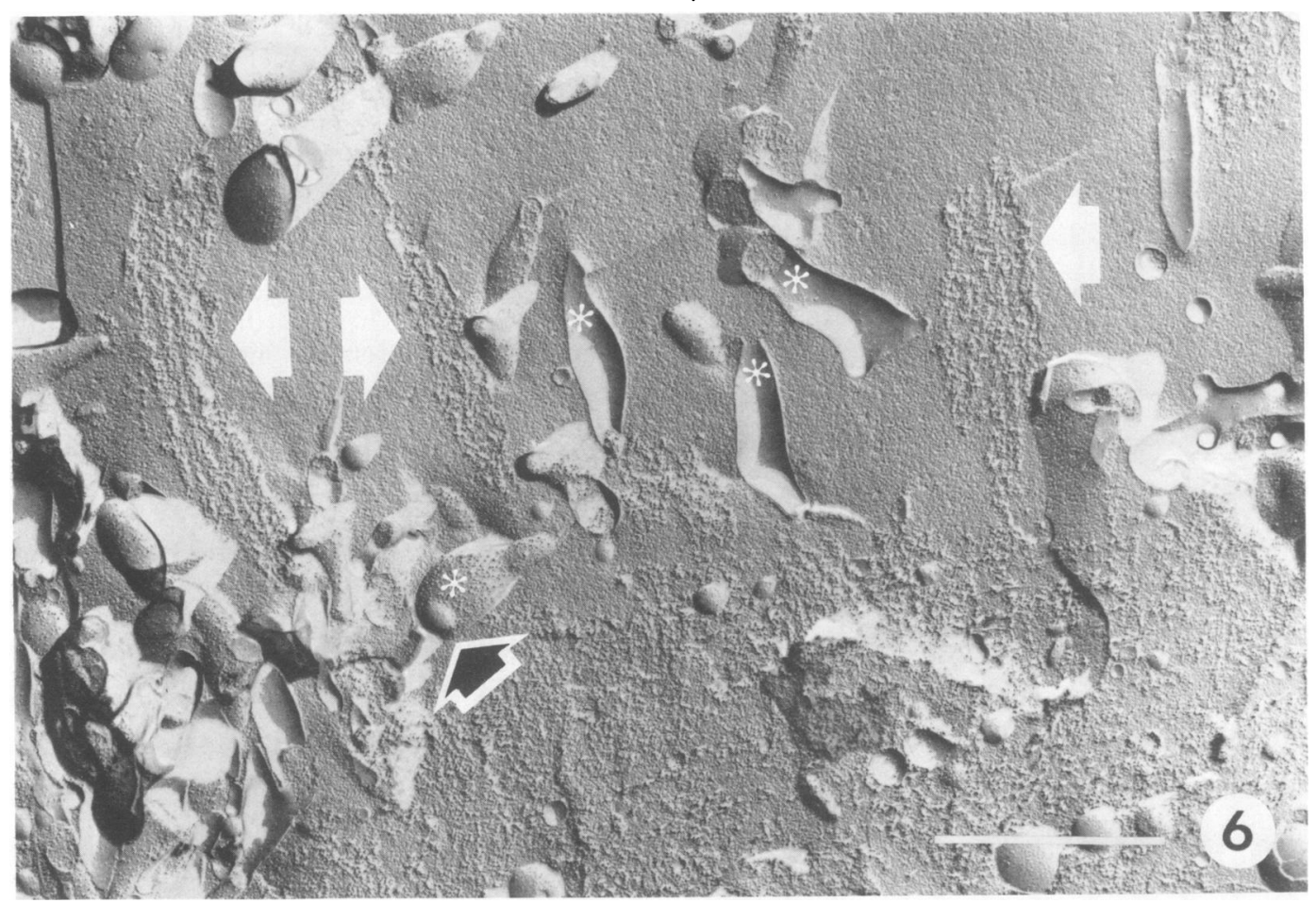

Fig. 6. A markedly deteriorated epithelial cell can be observed in this freeze-etch preparation of an infected tracheal organ culture after $48 \mathrm{~h}$ of incubation. The cytosol of the epithelial cell can be seen dissipating into the surrounding milieu (white arrows). Several mycoplasma cells $\left(^{*}\right)$ are visible, one of which is lying adjacent to a portion of the remaining epithelial cell membrane and illustrating a particle-free bleb on the hydrophobic face of the fractured membrane (black on white arrow). Bar, $1.0 \mu$ m.

cated by deteriorated ciliary necklaces and the formation of plaques of intramembranous particles on the shafts of the affected cilia. In addition to these previously reported observations (3), it was also noted that a disorganization and apparent misalignment of adjacent cilia may occur in experimental $M$. pneumoniae infection, suggesting a possible loss of synchrony in the ciliary beat.

With extended incubation time, the lumenal surface membranes of the infected epithelial cells were observed to disintegrate with the subsequent leakage of the cytosol from the cell. At this point, the histological integrity of the epithelial cells is lost and such cells probably represent dead epithelial cells, which subsequently slough away from the surface of the airway in both experimental and natural infection. Previous studies (13) have demonstrated that host cell ribonucleic acid and protein synthesis are altered within $24 \mathrm{~h}$ after infection of tracheal rings by virulent mycoplasmas. Thus the morphological alterations observed in infected host epithelial cells in these studies may represent a structural analog to the findings of prior biochemical studies.

Fig. 7. Micrograph from a freeze-etch preparation of a control uninfected tracheal organ culture after 48 $h$ of incubation. The black lines on the cilia indicate the parallel directional alignment of adjacent cilia in control organ cultures. The micrograph also reveals the normal structure of the ciliary necklaces (arrows) on several adjacent cilia. This complex in control hamster ciliated epithelial cells is comprised of four to six rows of evenly spaced membrane-associated particles. Note also the uniform distribution of membraneassociated particles on the shafts of the cilia. Bar, $0.1 \mu \mathrm{m}$.

Fig. 8. Micrograph from a freeze-etch preparation of an $M$. pneumoniae-infected tracheal organ culture after $48 \mathrm{~h}$ of incubation. The black lines on the cilia indicate an altered nonparallel directional alignment of adjacent cilia suggestive of asynchrony. The micrograph reveals the disorganized ciliary necklace on several adjacent cilia (black arrows) and plaques of membrane-associated particles on the ciliary shafts (white arrows). Bar, $0.1 \mu \mathrm{m}$. 

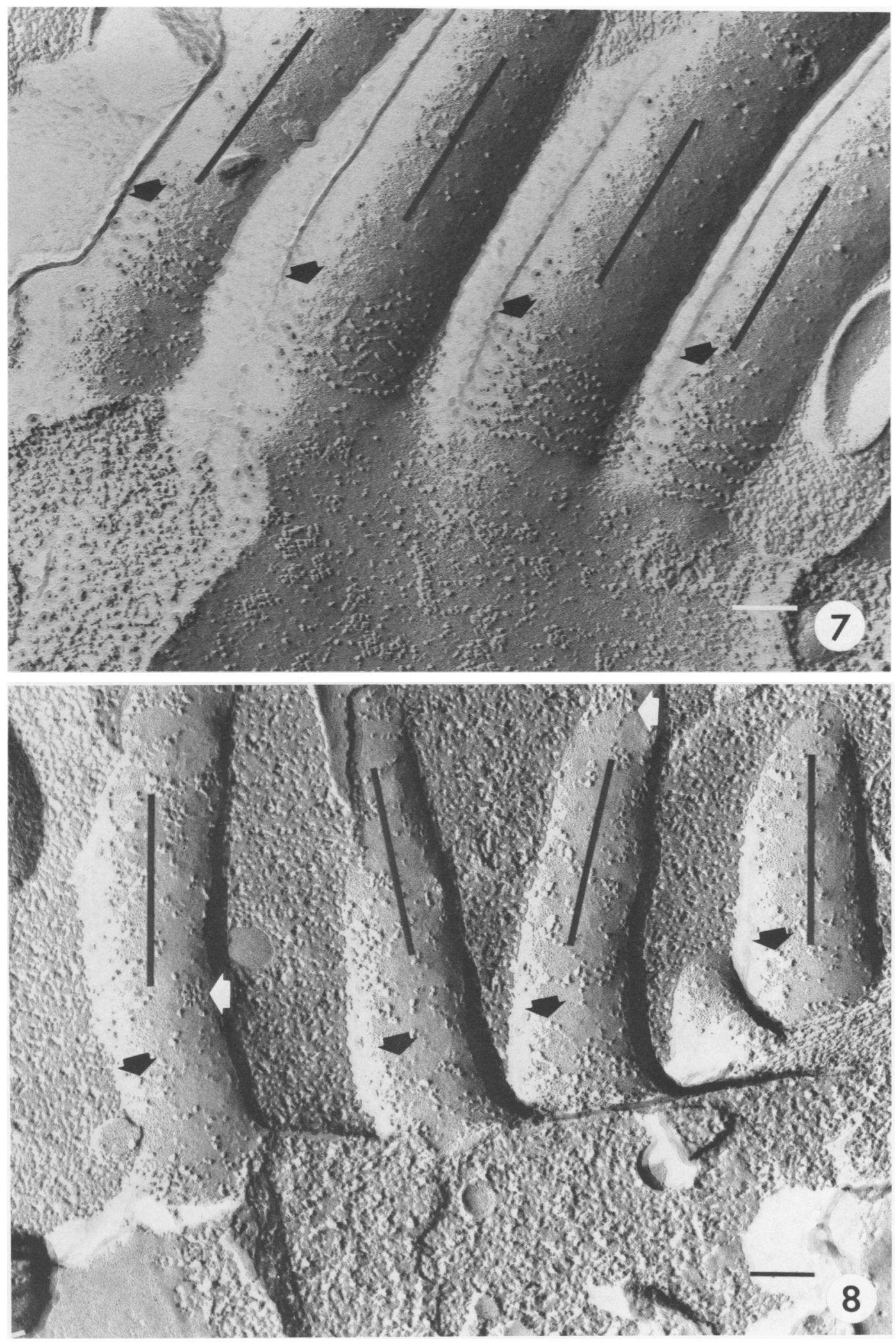
In summary, previous studies have contributed much valuable data regarding the internal ultrastructural organization and pathogenic mechanisms of $M$. pneumoniae. Our studies, utilizing an additional approach, have provided information concerning the structure of both host and pathogen membranes in experimental infection. The host-pathogen interactions observed in these studies may extend beyond morphological changes and reflect essential changes at the biochemical level, which affect the basic physiology and metabolism of the infected host epithelium.

\section{ACKNOWLEDGMENTS}

We thank Wallace A. Clyde, Jr., for his review and comments in the preparation of this manuscript.

This research was supported by SCOR grant HL19171 from the National Heart, Lung and Blood Institute and training grant Al07151 from the National Institute of Allergy and Infectious Diseases.

\section{LITERATURE CITED}

1. Bernstein-Ziv, R. 1969. Cell division in Mycoplasma gallisepticum. Can. J. Microbiol. 15:1125-1128

2. Branton, D. 1969. Membrane structure. Ann. Rev. Plant Physiol. 20:209-238.

3. Carson, J. L., A. M. Collier, W. A. Clyde, Jr. 1979. Ciliary membrane alterations occurring in experimental Mycoplasma pneumoniae infection. Science 206:349351.

4. Carson, J. L., A. M. Collier, and S. S. Hu. 1980. Ultrastructural studies of hamster tracheal epithelium in vivo and in vitro. J. Ultrastr. Res. 70:70-81.

5. Collier, A. M., and W. A. Clyde, Jr. 1971. Relationships between Mycoplasma pneumoniae and human respiratory epithelium. Infect. Immun. 3:694-701.

6. Collier, A. M., and J. B. Baseman. 1973. Organ culture techniques with mycoplasmas. Ann. N.Y. Acad. Sci. 225:277-289.

7. Collier, A. M., and W. A. Clyde, Jr. 1974. Appearance of Mycoplasma pneumoniae in lungs of experimentally infected hamsters and sputum from patients with natural disease. Am. Rev. Respir. Dis. 110:765-773.

8. Clyde, W. A., Jr. 1975. Pathogenic mechanisms in mycoplasma diseases, p. 143-146. In D. Schlessinger (ed.), Microbiology-1975. American Society for Microbiology, Washington, D. C.

9. Denny, F. W., W. A. Clyde, Jr., and W. P. Glezen. 1971. Mycoplasma pneumoniae disease: clinical spectrum, pathophysiology, epidemiology, and control. J. Infect. Dis. 123:74-92.

10. Gilula, N. B., and P. Satir. 1972. The ciliary necklace a ciliary membrane specialization. J. Cell. Biol. 53:494509.

11. Green, F., III, and R. P. Hanson. 1973. Ultrastructure and capsule of Mycoplasma meleagridis. J. Bacteriol. 116:1011-1018.

12. Hayflick, L. 1965. Tissue cultures and mycoplasmas. Tex. Rep. Biol. Med. 23:285-303.

13. Hu, P. C., A. M. Collier, and J. B. Baseman. 1975. Alterations in the metabolism of hamster tracheas in organ culture after infection by virulent Mycoplasma pneumoniae. Infect. Immun. 11:704-710.

14. Moor, H. 1966. Use of freeze-etching in the study of biological ultrastructure. Int. Rev. Exp. Pathol. 5:179216.

15. Muse, K. E., D. A. Powell, and A. M. Collier. 1976. Mycoplasma pneumoniae in hamster tracheal organ culture studied by scanning electron microscopy. Infect. Immun. 13:229-237.

16. Tillack, T. W., R. Carter, and S. Razin. 1970. Native and reformed Mycoplasma laidlawii membranes compared by freeze-etching. Biochim. Biophys. Acta 219: 123-130.

17. Tourtellote, M. E., and J. S. Zupnik. 1973. Freezefractured Acholeplasma laidlawii membranes: nature of particles observed. Science 179:84-86.

18. Wilson, M. H., and A. M. Collier. 1976. Ultrastructural study of Mycoplasma pneumoniae in organ culture. J. Bacteriol. 125:332-339. 